\title{
HOW TO BUILD VALID AND CREDIBLE SIMULATION MODELS
}

\author{
Averill M. Law \\ Averill M. Law \& Associates, Inc. \\ 6601 East Grant Road, Suite 110 \\ Tucson, AZ 85715, U.S.A.
}

\begin{abstract}
In this tutorial we present techniques for building valid and credible simulation models. Ideas to be discussed include the importance of a definitive problem formulation, discussions with subject-matter experts, interacting with the decision-maker on a regular basis, development of a written assumptions document, structured walk-through of the assumptions document, use of sensitivity analysis to determine important model factors, and comparison of model and system output data for an existing system (if any). Each idea will be illustrated by one or more real-world examples. We will also discuss the difficulty in using formal statistical techniques (e.g., confidence intervals) to validate simulation models.
\end{abstract}

\section{WHAT IS MODEL VALIDATION}

Use of a simulation model is a surrogate for experimentation with the actual system (existing or proposed), which is usually disruptive, not cost-effective, or simple impossible. Thus, if the model is not a "close" approximation to the actual system, any conclusions derived from the model are likely to be erroneous and may result in costly decisions being made. Validation should and can be done for all models, regardless of whether the corresponding system exists in some form or whether it will be built in the future.

We now give definitions of validation and credibility. Validation is the process of determining whether a simulation model is an accurate representation of the system, for the particular objectives of the study. The following are some general perspectives on validation:

- A "valid" model can be used to make decisions similar to those that would be made if it were feasible and cost-effective to experiment with the system itself.

- The ease or difficulty of the validation process depends on the complexity of the system being modeled and on whether a version of the system currently exists (see Section 2.8). For example, a model of a neighborhood bank would be relatively easy to validate since it could be closely observed. On the other hand, a model of the effectiveness of a naval weapons system in the year 2025 would be virtually impossible to validate completely, since the location of the battle and the nature of the enemy weapons would be unknown. Also, it is often possible to collect data on an existing system that can be used for building and validating a model.

- A simulation model of a complex system can only be an approximation to the actual system, no matter how much time and money is spent on model building. There is no such thing as absolute model validity, nor is it even desired. Indeed, a model is supposed to be an abstraction and simpli-fication of reality. The more time (and hence money) that is spent on model development, the more valid the model should be in general. How-ever, the most valid model is not necessarily the most costeffective. For example, increasing the validity of the model beyond a certain level might be quite expensive, since extensive data collection may be required, but might not lead to signifi-cantly better insight or decisions.

- A simulation model should always be developedfor a particular set of objectives. In fact, a model that is valid for one objective may not be for another.

- Validation is not something to be attempted after the simulation model has already been developed, and only if there is time and money remaining. Unfortunately, our experience indicates that this recommendation is often not followed.

Example 1. An organization paid a consulting company $\$ 500,000$ to perform a "simulation study" that had a six-month duration. After the study was supposedly completed, a person from the client organization called us and asked, "Can you tell me in five minutes on the phone how to validate our model?" 
- Each time a simulation model is being considered for a new application its validity needs to be reexamined. The current purpose may be substantially different from the original purpose or the passage of time may have invalidated certain model parameters.

A simulation model and its results have credibility if the decision-maker and other key project personnel accept them as "correct." Note that a credible model is not necessarily valid, and vice versa. The following things help establish credibility for a model:

- The decision-maker's understanding of and agreement with the model's assumptions

- Demonstration that the model has been validated and verified (i.e., that the model computer program has been debugged)

- The decision-maker's ownership of and involvement with the project

- Reputation of the model developers

- A compelling animation.

The U.S. Department of Defense (DoD) is a large user of simulation models, and in recent years years there has been considerable interest in verification, validation, and a concept known as accreditation (VV\&A). Accreditation (see Defense Modeling and Simulation Office 2000) is the official certification (by the project sponsor) that a simulation model is acceptable for a specific purpose. The main reason that accreditation is mandated within DoD is that someone must take the responsibility for the decision to use a model for a particular application, since a large amount of money and people's lives may be at stake.

Note that many of the ideas and examples presented in this paper are based on the chapter "Building Valid, Credible, and Appropriately Detailed Simulation Models" in (Law 2006) and also on the simulation short courses presented by the author. Other references on model validation are (Balci 1998), (Banks et al. 2005), (Carson 1986), (Sargent 2004), and (Shannon 1975).

The remainder of this paper is organized as follows. We present in Section 1.1 a seven-step approach for conducting a successful simulation study. In Section 2 we discuss techniques for developing a more valid and credible simulation model. Guidelines for obtaining good model data are given in Section 3. Finally, Section 4 provides a summary of the most important validation ideas.

\subsection{A Seven-Step Approach for Conducting a Success- ful Simulation Study}

In Figure 1 we present a seven-step approach for conducting a successful simulation study. Having a definitive approach for conducting a simulation study is critical to the study's success in general and to developing a valid model in particular. In Section 2 we will relate each of our validation/credibility enhancement techniques to one or more of these steps. We now discuss important activities that take place in each of the seven steps.

\section{Step 1. Formulate the Problem}

- Problem of interest is stated by the decisionmaker.

- It may not be stated precisely or in quantitative terms.

- An iterative process is often necessary.

- A kickoff meeting(s) for the simulation project is (are) conducted, with the project manager, the simulation analysts, and subject-matter experts (SMEs) in attendance. The following things are discussed at this meeting:

- The overall objectives for the study

- The specific questions to be answered by the study (without such specificity it is impossible to determine the appropriate level of model detail).

- The performance measures that will be used to evaluate the efficacy of different system configurations.

- The scope of the model

- The system configurations to be modeled

- The time frame for the study and the required resources (people, computers, etc.).

\section{Step 2. Collect Information/Data and Construct As- sumptions Document}

- Collect information on the system layout and operating procedures.

- Collect data to specify model parameters and probability distributions (e.g., for the time to fail-ure and the time to repair of a machine).

- Document the model assumptions, algorithms, and data summaries in a written assumptions document (sometimes called a conceptual model).

- The level of model detail should depend on the following:

- Project objectives .

- Performance measures of interest.

- Data availability.

- Credibility concerns.

- Computer constraints.

- Opinions of SMEs.

- $\quad$ Time and money constraints.

- There should not be a one-to-one correspondence between the model and the system. 


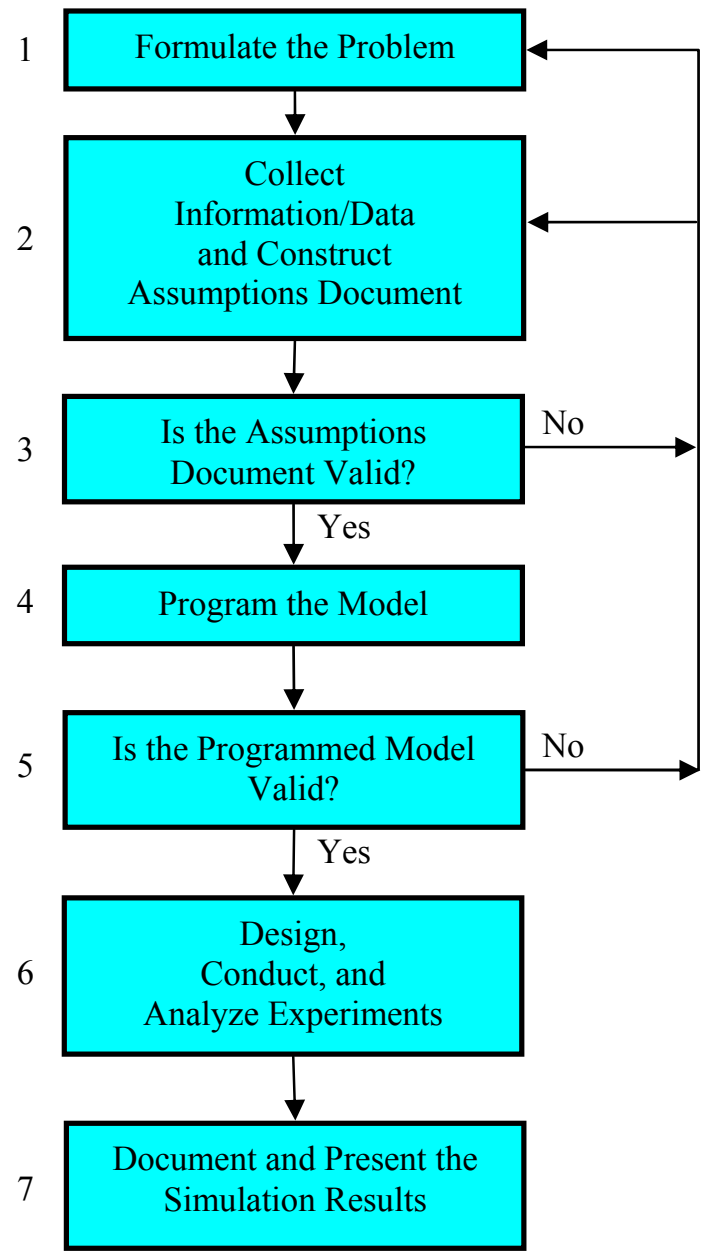

Figure 1: A Seven-Step Approach for Conducting a Successful Simulation Study

- Collect performance (output) data from the existing system (if any) to use for model validation in Step 5.

\section{Step 3. Is the Assumptions Document Valid?}

- Perform a structured walk-through of the assumptions document before an audience that includes the project manager, analysts, and SMEs. Within the DoD community, this is sometimes called conceptual-model validation.

- If errors or omissions are discovered in the assumptions document, which is almost always the case, then the assumptions document must be updated before proceeding to programming in Step 4.

\section{Step 4. Program the Model}

- Program the assumptions document in a commercial simulation-software package or in a generalpurpose programming language (e.g., $\mathrm{C}, \mathrm{C}++$, and Java).

- Verify (debug) the computer program.

\section{Step 5. Is the Programmed Model Valid?}

- If there is an existing system, then compare simulation model output data for this system with the comparable output data collected from the actual system (see Step 2). This is called results validation.

- Regardless of whether there is an existing system, the simulation analysts and SMEs should review the simulation results for reasonableness. If the results are consistent with how they perceive the system should operate, then the simulation model is said to have face validity.

- Sensitivity analyses should be performed on the programmed model to see which model factors have the greatest impact on the performance measures and, thus, have to be modeled carefully.

\section{Step 6. Design, Conduct, and Analyze Experiments}

- For each system configuration of interest, decide on tactical issues such as run length, warmup period, and the number of independent model replications.

- Analyze the results and decide if additional experiments are required.

\section{Step 7. Document and Present the Simulation Results}

- The documentation for the model (and the associated simulation study) should include the assumptions document (critical for future reuse of the model), a detailed description of the computer program, and the results of the current study.

- The final presentation for the simulation study should include an animation and a discussion of the model building/validation process to promote model credibility.

\section{TECHNIQUES FOR DEVELOPING VALID AND CREDIBLE MODELS}

In this section we present practical techniques for developing valid and credible models. At the end of each subsection title, we state in square brackets ("[ ]") in which of the seven steps (at a minimum) the technique should be applied. 


\subsection{Formulating the Problem Precisely [1]}

It is critical to formulate the problem of interest in a precise manner. This should include an overall statement of the problem to be solved, a list of the specific questions that the model is to answer, and the performance measures that will be used to evaluate the efficacy of particular system configurations. Without a definitive statement of the specific questions of interest, it is impossible to decide on an appropriate level of model detail. Performance measures must also be clearly stated since different measures may dictate different levels of model detail; see (Law 2006), pp. 687-688 for an example.

When the decision-maker first initiates a simulation study, the exact problem to be solved is sometimes not precisely stated or even completely understood. Thus, as the study proceeds and a better understanding is obtained, this information should be communicated to the decisionmaker who may reformulate the problem.

\subsection{Interviewing Subject-Matter Experts [1, 2]}

There will never be a single person who knows all of the information necessary to build a simulation model. Thus, it will be necessary for the simulation analysts to talk to many different SMEs to gain a complete understanding of the system to be modeled. Note that some of the information supplied by the SMEs will invariably be incorrect - if a certain part of the system is particularly important, then at least two SMEs should be queried. In Section 2.6, we will discuss a technique that helps ensure that a model's assumptions are correct and complete - this technique is also useful for resolving differences of opinion among SMEs.

\subsection{Interacting with the Decision-Maker on a Regular Basis [1-7]}

One of the most important ideas for developing a valid and credible model is for the analyst to interact with the decision-maker and other members of the project team on a regular basis. This approach has the following key benefits:

- Helps ensure that the correct problem is solved.

- The exact nature of the problem may not be initially known.

- The decision-maker may change his/her objectives during the course of the study.

- The decision-maker's interest and involvement in the study are maintained.

- The model is more credible because the decisionmaker understands and agrees with the model's assumptions.
Example 2. A military analyst worked on a simulation project for several months without interacting with the general who requested it. At the final Pentagon briefing for the study, the general walked out after five minutes stating, "That's not the problem I'm interested in."

\subsection{Using Quantitative Techniques to Validate Com- ponents of the Model [2]}

The simulation analyst should use quantitative techniques whenever possible to test the validity of various components of the overall model. We now give examples of techniques that have been used for this purpose.

If one has fit a theoretical probability distribution (e.g., exponential or normal) to a set of observed data, then the adequacy of the representation can be assessed by using graphical plots and goodness-of-fit tests; see (Law 2006), chapter 6.

As will be discussed in Section 3, it is important to use appropriate data in building a model; however, it is equally important to exercise care when structuring these data. For example, if several sets of data have been observed for the "same" random phenomenon, then the correctness of merging these data sets can be assessed by using the KruskalWallis test of homogeneity of populations; see (Law 2006), pp. 380-381. If the data sets appear to be homogeneous, they can be merged and the combined data set used for some purpose in the simulation model.

Example 3. Consider a manufacturing system for which time-to-failure and time-to-repair data were collected for two "identical" machines made by the same vendor. However, the Kruskal-Wallis test showed that the two distributions were, in fact, different for the two machines. Thus, each machine was given its own timeto-failure and time-to-repair distributions in the simulation model.

\subsection{Documenting the Assumptions Document [2]}

Communication errors are a major reason why simulation models very often contain invalid assumptions. The documentation of all concepts, assumptions, algorithms, and data summaries can lessen this problem. It will also increase the credibility of the model. An assumptions document should not be an "exact" description of how the system works, but rather a description of how it works relative to the particular issues that the model is to address. This report is the major documentation for the model and should be readable by analysts, SMEs, and technical managers. The following are some of the things that should be included in the assumptions document: 
- An overview section that discusses overall project goals, specific issues to be addressed by the model, and relevant performance measures.

- A process-flow/system-layout diagram (if appropriate).

- Detailed descriptions of each subsystem (in bullet format for easy reading) and how they interact.

- What simplifying assumptions were made and why.

- Limitations of the model.

- Summaries of model input data (technical analyses should be put in appendices to promote report readability by decision-makers).

- Sources of important or controversial information.

The assumptions document should contain enough detail so that it is a "blueprint" for creating the simulation computer program (in Step 4).

\subsection{Performing a Structured Walk-through of the As- sumptions Document [3]}

As previously discussed, the simulation analyst will need to collect system information from many different SMEs. Furthermore, these people are typically very busy dealing with the daily problems that occur within their organization, often resulting in their giving something less than their undivided attention to the questions posed by the simulation analyst. As a result, there is a considerable danger that the analyst will not obtain a complete and correct description of the system. An effective way of dealing with this potential problem is to conduct a structured walkthrough of the assumptions document before an audience of SMEs and decision-makers. Using a projection device, the analyst goes through the assumptions document bulletby-bullet, but not proceeding from one bullet to the next until everybody in the room is convinced that a particular bullet is correct and at an appropriate level of detail. A structured walk-through will increase both the validity and credibility of the simulation model. (As stated above, this exercise is sometimes called conceptual-model validation.)

The structured walk-through should ideally be held at a remote site (e.g., a hotel meeting room), so that people give the meeting their full attention. Furthermore, it should be held prior to the beginning of programming in case major problems are uncovered at the meeting. The assumptions document should be sent to participants prior to the meeting and their comments requested. We do not, however, consider this a substitute for the structured walkthrough itself, since people may not have the time or motivation to review the document carefully on their own. Furthermore, the interactions that take place at the actual meeting are invaluable.
Example 4. At a structured walk-through of a transportation system, a significant percentage of the assumptions given to us by our corporate sponsor were found to be wrong by the SMEs present. (Due to the large geographic distances between the home offices of the sponsor and the SMEs, it was not possible for the SMEs to be present at the kickoff meeting for the project.) As a result, various people were assigned responsibilities to collect information on different parts of the system. The collected information was used to update the assumptions document, and a second walkthrough was successfully performed.

\subsection{Performing Sensitivity Analyses to Determine Im- portant Model Factors [5]}

An important technique for determining which model factors have a significant impact on the desired measures of performance is sensitivity analysis. If a particular factor appears to be important, then it needs to be modeled carefully. The following are examples of factors that could be investigated by a sensitivity analysis:

- The value of a parameter (see Example 5).

- The choice of a probability distribution.

- The entity moving through the simulated system.

- The level of detail for a subsystem.

Example 5. In a simulation study of a new system, suppose that the value of a probability is estimated to be 0.75 as a result of conversations with SMEs. The importance of getting the value of this probability "exactly" correct can be determined by running the simulation with 0.75 and, for example, by running it with each of the values 0.70 and 0.80 . If the three simulation runs produce approximately the same results, then the output is not sensitive to the choice of the probability over the range 0.70 to 0.80 . Otherwise, a better specification of the probability is needed. (Strictly speaking, to determine the effect of the probability on the model's results, we should make several independent replications of the model using different random numbers for each of the three cases.)

If one is trying to determine the sensitivity of the simulation output to changes in two or more factors of interest, then it is not correct, in general, to vary one factor at a time while setting the other factors at some arbitrary values. (This dangerous practice is sometimes called the onefactor-at-a-time approach.) A more correct approach is to use statistical experimental design, which is discussed in (Law 2006, Chapter 12) and in (Montgomery 2005). The effect of each factor can be formally estimated and, if the number of factors is not too large, interactions among the factors can also be detected. 


\subsection{Validating the Output from the Overall Simula- tion Model [5]}

The most definitive test of a simulation model's validity is establishing that its output data closely resemble the output data that would be observed from the actual system. If a system similar to the proposed one now exists, then a simulation model of the existing system is developed and its output data are compared to those from the existing system itself. If the two sets of data compare "closely," then the model of the existing system is considered "valid." (The accuracy required from the model will depend on its intended use and the utility function of the decisionmaker.) The model is then modified so that it represents the proposed system. The greater the commonality between the existing and proposed systems, the greater our confidence in the model of the proposed system. There is no completely definitive approach for validating the model of the proposed system. If there were, then there might be no need for a simulation model in the first place. If the above comparison is successful, then it has the additional benefit of providing credibility for the use of simulation. The comparison of the model and system output data could be done using numerical statistics such as the sample mean, the sample variance, and the sample correlation function. Alternatively, the assessment could be made by using graphical plots such as histograms, distribution functions, and box plots. (As stated above, the idea of comparing the model and system output data for the existing system is called results validation.)

Example 6. A U.S. Air Force test agency performed a simulation study for a bomb wing of bombers using the Logistics Composite Model (LCOM). The ultimate goal of the study was to evaluate the effect of various proposed logistics policies on the availability of the bombers, i.e., the proportion of time that the bombers were available to fly missions. Data were available from the actual operations of the bomb wing over a 9month period, and included both failure data for various aircraft components and a bomb-wing availability of 0.9. To validate the model, the Air Force first simulated the 9-month period with the existing logistics policy and obtained a model availability of 0.873 , which is 3 percent different than the historical availability. This difference was considered acceptable because an availability of 0.873 would still allow enough bombers to be available for the Air Force to meet its mission requirements.

Example 7. A manufacturer of heat-treated aluminum products was thinking of replacing its existing batch furnace by a new continuous furnace in order to increase its production capacity (see Law 1991). We first simulated the existing system and found that the model monthly throughput differed from the historical monthly throughput by less than one percent. Thus, it appeared that the model of the existing system was reasonably "valid."

A number of statistical tests ( $t$, Mann-Whitney, etc.) have been suggested in the validation literature for comparing the output data from a simulation model with those from the corresponding real-world system (see, for example, Shannon 1975, p. 208). However, the comparison is not as simple as it might appear, since the output processes of almost all real-world systems and simulations are nonstationary (the distributions of the successive observations change over time) and autocorrelated (the observations in the process are correlated with each other). Thus, classical statistical tests based on independent, identically distributed (IID) observations are not directly applicable. Furthermore, we question whether hypothesis tests, as compared with constructing confidence intervals for differences, are even the appropriate statistical approach. Since the model is only an approximation to the actual system, a null hypothesis that the system and model are the "same" is clearly false. We believe that it is more useful to ask whether or not the differences between the model and the system are significant enough to affect any conclusions derived from the model. For a brief discussion of statistical procedures that can be used to compare model and system output data, see Section 2.10.

In addition to statistical procedures, one can use a Turing test (Schruben 1980) to compare the output data from the model to those from the system. People knowledgeable about the system (e.g., SMEs) are asked to examine one or more sets of system data as well as one or more sets of model data without knowing which data sets are which. If these SMEs can differentiate between the system and model data, their explanation of how they were able to do so is used to improve the model.

Example 8. An animation version of the Turing test was used in validating a simulation model of microscopic vehicle flow on a freeway. An animation of traffic flow from the simulation was displayed simultaneously on a large-screen monitor with an animation produced from data collected from the actual freeway. The data from the freeway were collected by a video camera mounted on an airplane.

Whether or not there is an existing system, analysts and SMEs should review simulation output (numerical results, animations, etc.) for reasonableness. (Care must be taken in performing this exercise, since if one knew exactly what output to expect, then there would be no need for a model.) If the simulation results are consistent with perceived system behavior, then, as stated above, the model is said to have face validity. 
Example 9. The above idea was put to good use in the development of a simulation model of the U.S. Air Force manpower and personnel system. (This model was designed to provide Air Force policy analysts with a system-wide view of the effects of various proposed personnel policies.) The model was run under the baseline personnel policy, and the results were shown to Air Force analysts and decision-makers, who subsequently identified some discrepancies between the model and perceived system behavior. This information was used to improve the model, and after several additional evaluations and improvements, a model was obtained that appeared to approximate current Air Force policy closely. This exercise improved not only the validity of the model, but also its credibility.

Suppose than another model was developed for the same system and for a similar purpose, and that it is thought to be a "valid" representation. Then numerical statistics and, graphical plots can be compared for the two models. It should be noted that just because two models produce similar results doesn't necessarily mean that either model is valid, since both models could contain a similar error. This idea seems to be the most-widely used for defense applications.

\subsection{Animation [5-7]}

An animation is useful for showing that a simulation model is not valid and for promoting model credibility. It is also valuable for verification of the simulation computer program, for suggesting improved operational procedures, and for training.

\subsection{Statistical Techniques for Comparing Model and System Output Data [5]}

In this section we discuss the possible use of statistical procedures for carrying out the comparison of model and system output data discussed in Section 2.8.

Suppose that $R_{1}, R_{2}, \ldots, R_{k}$ are observations from a real-world system and that $M_{1}, M_{2}, \ldots, M_{l}$ are output data from a corresponding simulation model (see Example 10 below). We would like to compare these data sets in some way to determine whether the model is an accurate representation of the real-world system. However, most classical statistical approaches such as confidence intervals and hypothesis tests assume that the real-world data and the model data are each IID data sets, which is generally not the case (see the discussion in Section 2.8). Thus, these classical statistical approaches are not directly applicable to our comparison problem.
Example 10. Consider a manufacturing system where the output data of interest are the times in system of successively completed parts. These data are not independent for the actual system (nor for a corresponding simulation model). For example, if the system is busy at a particular point in time, then all of the parts being processed will tend to have large times in system (i.e., the times are positively correlated).

Law (2006, pp. 265-273) discusses inspection, confidence-interval, and time-series approaches that might possibly be used for comparing model and system output data.

\section{GUIDELINES FOR OBTAINING GOOD MODEL DATA}

A model is only valid for a particular application if its logic is correct and if it uses appropriate data. In this section we provide some suggestions on how to obtain good model data.

\subsection{Two Basic Principles}

If a system similar to the one of interest exists, then data should be obtained from it for use in building the model. These data may be available from historical records or may have to be collected during a time study. Since the people who provide the data might be different from the simulation analysts, it is important that the following two principles be followed:

- The analysts need to make sure that the data requirements (type, format, amount, why needed, conditions under which it should be collected, etc.) are specified precisely to the people who provide the data.

- The analysts need to understand the process that produced the data, rather than treating the observations as just abstract numbers. For example, suppose that data are available on the time to load a ship, but there are a few observations that are significantly larger than the rest (called outliers). Without a good understanding of the underlying process, it is impossible to know whether these large observations are the result of measuring or recording errors, or are just legitimate values that occur with small probability.

\subsection{Common Difficulties}

The following are four potential difficulties with data:

- Data are not representative of what one really wants to model. 
Example 11. The data that have been collected during a military field test may not be representative of actual combat conditions due to differences in troop behavior and to lack of battlefield smoke.

- Data are not of the appropriate type or format.

Example 12. In modeling a manufacturing system, the largest source of randomness is usually random downtimes of a machine. Ideally, we would like data on time to failure (in terms of actual machine busy time) and time to repair of a machine. Sometimes data are available on machine breakdowns, but quite often they are not in the proper format. For example, the times to failure might be based on wall-clock time and include periods that the machine was idle or off-shift.

- Data may contain measuring, recording, or rounding errors.

Example 13. Data representing the time to perform some task are sometimes rounded to the closest 5 or 10 minutes. This may make it difficult to fit a continuous theoretical probability distribution to the data, since the data are now discrete.

- Data may be "biased" because of self-interest.

Example 14. The maintenance department in an automotive factory reported the reliability of certain machines to be greater than reality to make themselves look good.

\section{SUMMARY}

All simulation models need to be validated or any decisions made with the model may be erroneous. The following are the ideas that we believe are the most important for developing a valid and credible model:

- $\quad$ Formulating the problem precisely.

- Interviewing appropriate SMEs.

- Interacting with the decision-maker on a regular basis throughout the simulation project to ensure that the correct problem is being solved and to promote model credibility.

- Using quantitative techniques to validate components of the model.

- Developing a written assumptions document.

- Performing a structured walk-through of the assumptions document - for a nonexistent system, this may be the single most-important validation technique.

- Performing sensitivity analyses to determine important model factors.
- Comparing model and system results for an existing system (if any) - this is, in general, the most definitive validation technique available.

- Using a Turing test to compare model and system output data.

- Reviewing of model results and animations to see if they appear to be reasonable.

- Comparing model output data with the comparable output data for another model that is thought to be "valid."

Many of the above ideas would seem to be just common sense. However, our experience indicates that they are very often not applied.

\section{REFERENCES}

Balci, O. 1998. Verification, validation, and testing. In The handbook of simulation, ed. J. Banks, Chapter 10. New York: John Wiley.

Banks, J., J. S. Carson, B. L. Nelson, and D. M. Nicol. 2005. Discrete-event system simulation. 4th ed. Upper Saddle River, N. J: Prentice-Hall.

Carson J. S., June 1986. Convincing users of model's validity is challenging aspect of modeler's job. Industrial Engineering 18: 74-85.

Defense Modeling and Simulation Office. 2000. VV\&A recommended practices guide. Millennium Edition. http: / /vva.dmso.mil. Alexandria, Virginia.

Law, A. M. July 1991. Simulation study puts the right heat on at Kaiser Aluminum. Industrial Engineering 23: 16-17.

Law, A. M. 2006. Simulation modeling and analysis. 4th ed. New York: McGraw-Hill.

Montgomery, D.C. 2005. Design and analysis of experiments. 6th ed. New York: John Wiley.

Sargent, R. G. 2004. Verification and validation of simulation models. In Proceedings of the 2004 winter simulation conference. Washington, D.C., 17-28. Piscataway, New Jersey: Institute of Electrical and Electronics Engineers.

Schruben, L.W. 1980. Establishing the credibility of simulations. Simulation 34: 101-105.

Shannon, R. E. 1975. Systems simulation: The art and science. Englewood Cliffs, N.J.: Prentice-Hall.

\section{AUTHOR BIOGRAPHY}

AVERILL M. LAW is President of Averill M. Law \& Associates, a company specializing in simulation training, consulting, and software. He has been a simulation consultant to numerous organizations including Accenture, ARCO, Boeing, Defense Modeling and Simulation Office, Hewlett-Packard, Kimberly-Clark, M\&M/Mars, Navy Modeling and Simulation Office, 3M, U.S. Air Force, and 
U.S. Army. He has presented more than 415 simulation short courses in 17 countries. He has written or coauthored numerous papers and books on simulation, operations research, statistics, manufacturing, and communications networks. This includes the book Simulation Modeling and Analysis, which is widely considered the "bible" of the simulation industry, with more than 100,000 copies in print. He developed the ExpertFit distribution-fitting software and also several videotapes on simulation modeling. He has been the keynote speaker at simulation conferences worldwide. He wrote a regular column on simulation for Industrial Engineering magazine. He has been a tenured faculty member at the University of Wisconsin-Madison and the University of Arizona. He has a Ph.D. in Industrial Engineering and Operations Research from the University of California at Berkeley. His e-mail address is <averillasimulation.ws> and his Web site is <http: / / www . averill-law. com>. 